\title{
Los bosques de la RAAS veinte años después del huracán Joan
}

\author{
Gladys Luna \\ (URACCAN)
}

Me da mucho gusto ver tantas caras conocidas, ya que hace rato que no nos mirábamos; y más gusto porque nos encontramos justo hablando de este tema que es la recuperación del bosque. Estamos hablando de estudiantes que participaron en la toma de datos que vamos a estar presentando los resultados hoy. Y estoy contenta por eso. También estoy contenta, y quiero felicitar por esto a Álvaro Rivas, director de la revista Wani, por este número que elaboraron para este evento, en donde están sintetizados los resultados de estos veinte años de esfuerzo que hizo la universidad de Michigan, pero también un montón de colaboradores y una gran cantidad de tesis de licenciaturas, maestrías y doctorados que generó este tema. Y la participación de las universidad URACCAN y BICU, en Bluefields, cuyos estudiantes participaron anualmente en las mediciones y toma de datos; el Centro de Investigaciones y Documentación de la Costa Atlántica, que jugó un papel muy importante para que estas investigaciones fueran posibles; la Universidad de Michigan y Hood College, auspiciadas por la Fundación Nacional de Ciencias (NSF), de los Estados Unidos, a través del Dr. John Vandermeer que es el actor principal de esta beca.

Soy ecóloga y toda mi presentación está sometida a los sentimientos ecológicos de cómo nosotros, a partir de la ecología, podemos de manera general tratar de entender cómo funciona el ecosistema. Y si entendemos cómo funcionan los ecosistema podemos aprovechar los recursos de esos ecosistemas, pero si no los entendemos se nos hace un poquito más complicado trabajar de una forma amigable con ellos.

Sabemos que esta afirmación no es nada nuevo: que los ecosistemas tropicales son los sistemas más diversos y complejos que tenemos en el mundo, junto a los ecosistemas de arrecifes. Los ecosistemas terrestres son los más complejos y diversos; y por encontrarse en una situación de equilibrio están sometidos a constantes perturbaciones, perturbaciones de todo tipo, no solamente de huracanes, también de incendios, deslizamientos, tormentas, entre otros. Y estos cambios están generando alteraciones en la composición, entre otras cosas, de la composición vegetal.

\section{Equilibrio y teoría de Competencia}

Actualmente, la ecología moderna está replanteándose la forma en que estas perturbaciones están sometiendo a los bosques tropicales, en el sentido de que antes se pensaba que una perturbación está causando un daño al ecosistema vegetal. Ahora entendemos que las perturbaciones son parte de la dinámica de la naturaleza y que son necesarias muchas veces para mantener esta alta diversidad, esta alta productividad de los sistemas ecológicos, en este caso en particular, de los bosques tropicales húmedos. El efecto de una perturbación puede abarcar todos los niveles de intensidad: desde aquellas que están causando perturbaciones catastróficas que pueden provocar grandes cambios en la composición de una comunidad perturbada, hasta otras que pueden ser imperceptibles, que no están cambiando sensiblemente la composición y estructura de una comunidad ecológica perturbada. Pero qué pasa con las perturbaciones que tienen niveles intermedios, bueno, intermedio fue el nombre con que se denominó a las perturbaciones del huracán Joan.

La teoría de competencia, que es una de las grandes teorías conocidas, que es cuando tenemos ciertas especies que están compitiendo por un mismo recurso, en este caso los árboles que están consumiendo todos carbón, nitrógeno, fósforo, etcétera, van a estar peleando por estos mismos recursos. Esto no pasa solo con los árboles, pasa con todas las especies. Si tenemos cinco platos de comida aquí, para todos nosotros, vamos a estar peleando por esos platos de comida. Y qué va a pasar, solamente comerán unos pocos fuertes y los más débiles se quedarán sin comer. Pues lo mismo pasa con las plantas. Estos sistemas, a partir de esta teoría de competencia, si no tuvieran una perturbación a lo largo de unos doscientos, trescientos años, tendríamos un bosque dominado por una o dos especies en el mejor de los casos. Pero eso no ocurre gracias a que cuando el proceso de competencia es muy fuerte y viene esta 
perturbación, elimina a estas especies que están dominando o codominando el ecosistema, para reiniciar nuevamente el proceso de competencia. Esto es lo que se llama la teoría de la competencia.

Por lo tanto, a partir de esta premisa, doctores como el señor John Vandermeer están afirmando que las perturbaciones catastróficas juegan un papel determinante en la preservación de la biodiversidad, porque están eliminando esta dominancia de unas pocas especias. En particular, la región caribeña está sometida a estas perturbaciones que son los huracanes, dada la frecuencia con que están llegando a la costa, en tierra firme. Aquí, el huracán Joan, que azotó la zona de Bluefields y sus alrededores para 1988, tenía la categoría 4 en la escala Simpson.

\section{El huracán Joan}

\begin{tabular}{|c|c|c|c|c|c|}
\hline Categoría & $\begin{array}{c}\text { Vientos } \\
\mathbf{( K m} / \mathbf{h})\end{array}$ & Vientos (mph) & $\begin{array}{c}\text { Marejada } \\
\text { (pies) }\end{array}$ & Daños & Nombre \\
\hline $\mathbf{4}$ & $210-249$ & $131-155$ & $13-18$ & Severos & Joan 1988 \\
\hline
\end{tabular}

primarios de bajura, como fue en el caso de la RAAS que tuvo un duración del 10 hasta el 23 de octubre. Después de la perturbación del Joan encontramos una zona de 500,000 ha de bosques devastados. Un panorama muy triste. Pero tenemos la dicha de saber que actualmente esta situación cambió. Pero éste era el panorama: no había ni una sola hoja en los árboles, el daño físico fue exageradamente grande, y eso preocupó a los directivos y a los trabajadores del Instituto de Recursos Naturales (IRENA) en ese entonces.

\section{Características del huracán Joan}

INICIO DE LAS INVESTIGACIONES

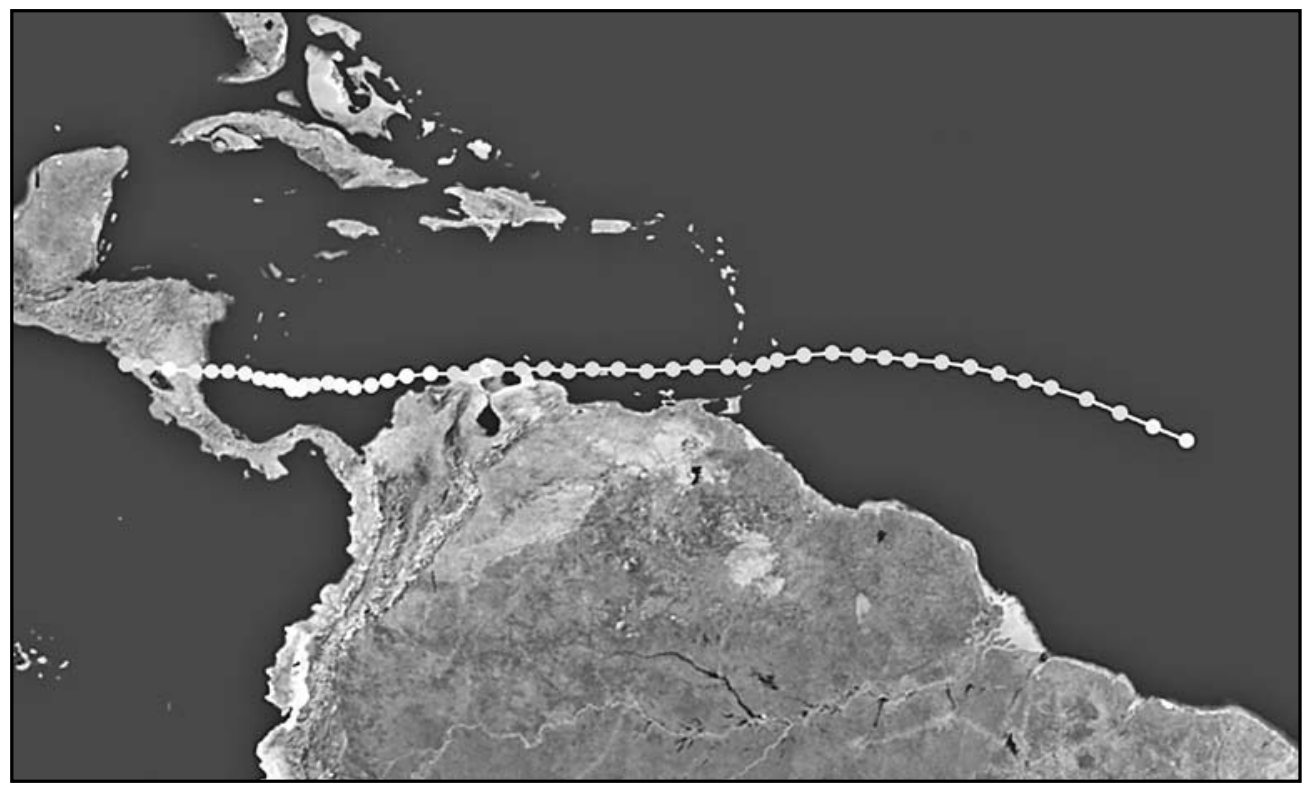

Fue entonces que IRENA hizo un llamado a la universidad de Michigan, para que viniera a estudiar este bosque. La universidad de Michigan llama entonces a investigadores de Hood Collage, y también hace contactos aquí en Nicaragua con la universidad Centroamericana (UCA). Así fue como nosotros y el CIDCA entramos a este proyecto. Entonces, este grupo de instituciones hicieron una primera expedición y el objetivo era cuantificar los daños que había ocasionado una perturbación de esta magnitud, y determinar cuáles

En la imagen que les estoy presentando tenemos la trayectoria que tuvo el huracán Joan, desde cuando se formó como una tormenta tropical, el 10 de octubre, hasta que llegó a su clímax al encontrarse con Bluefields el 22 de ese mes, por lo que causó todo un impacto puesto que llevaba vientos entre los 230 y $240 \mathrm{~km} / \mathrm{h}$.

Antes de 1989, el bosque impactado por el Joan fue un bosque primario, con un sotobosque dominado por fauna, muchas enredaderas, árboles muy altos, entre 25 y 30 $\mathrm{m}$, que es la altura característica del dosel de bosques serían las rutas de regeneración que seguiría el bosque.

\section{Metodología}

Fue así que para 1989, cuatro meses después de que ocurrió el huracán, se comienza a establecer las parcelas permanentes de muestreo en un bosque prácticamente tumbado, en el suelo, donde antes había árboles de 30 $\mathrm{m}$ de altura y que ahora se caminaba sobre ese dosel que estaba ya en el piso. 
UBICACIÓN DE LOS TRANSECTOS

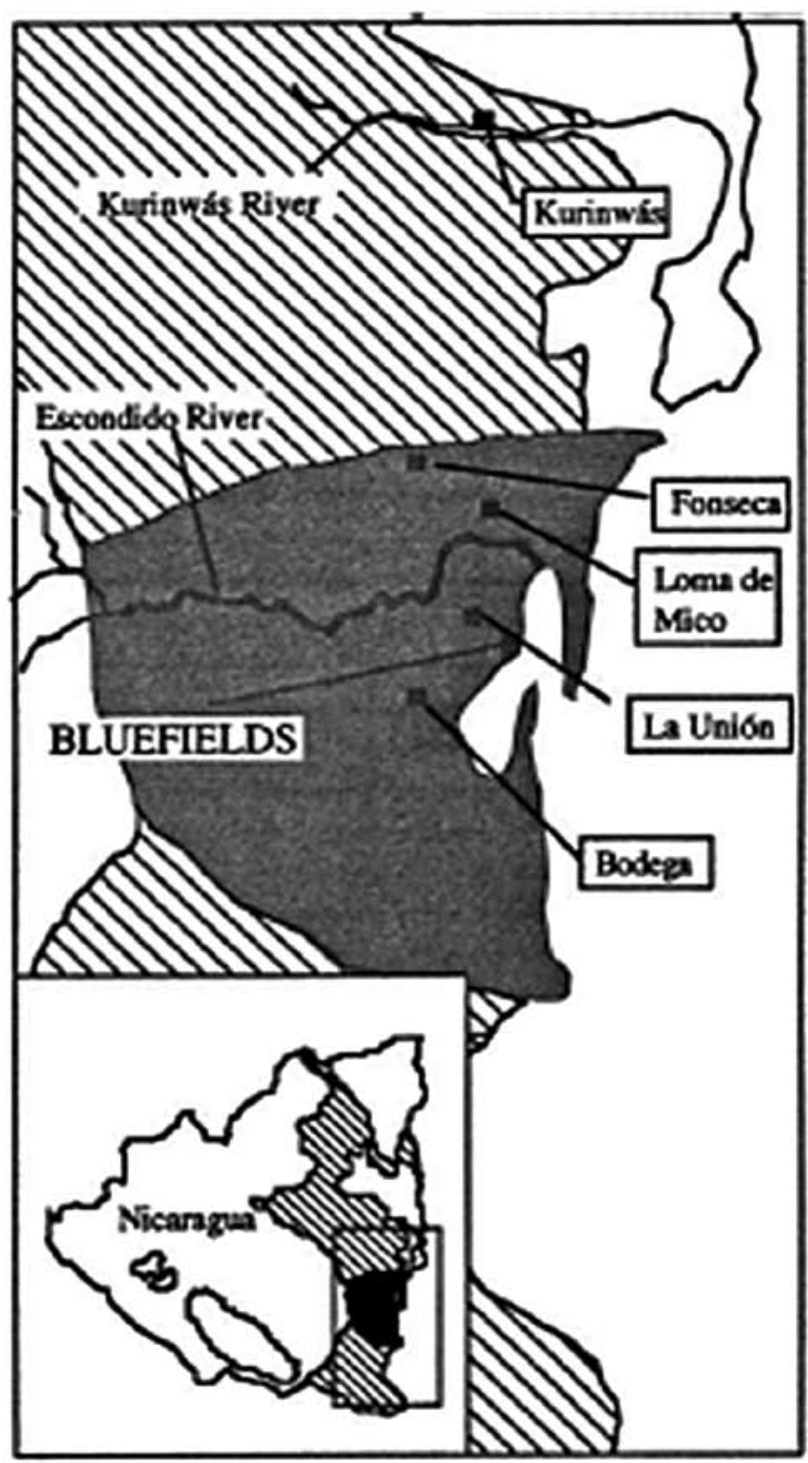

Fig. 2. Locaico of saxty sirex, area of incencive drmage from Hurtionse loan (shaded area) and extene of odiginal bowland rain foces (hached area).

Pero cuatro meses después cambia el panorama. Encontramos un bosque no recuperado, pero con mucho verde. Esto fue un aliciente para los costeños: saber que el bosque se estaba recuperando relativamente rápido. Se establecieron entonces parcelas permanentes de muestreo. Al inicio, para 1989, se establecieron únicamente dos: La Bodega, que después fue quemado, y La Fonseca. La Bodega queda a unos $14 \mathrm{~km}$ al sur de la trayectoria del ojo del huracán, y la Fonseca queda a unos $30 \mathrm{~km}$ al norte. Estas fueron las dos primeras parcelas de muestreo que se establecieron, que tenían un tamaño de 100 x $10 \mathrm{~m}$ y las siguientes parcelas se establecieron para 1994, cuando se tenía un poco más de fondos.
Conseguido estos fondos se establecieron las parcelas de La Unión y Caño Negro, que queda a $9 \mathrm{~km}$ al norte de la trayectoria del huracán, y Loma del Mico a $23 \mathrm{~km}$ al sur. Este lugar llamado La Bodega es por donde pasaron más cerca las hélices del huracán. Hay que saber que el ojo del huracán no afecta tanto como sus hélices. Entonces es allí donde se registró el mayor impacto físico del huracán. Para 1996 se establecen otras dos parcelas más: en Kurinwas, que queda muy, muy al Norte, a unos 100 km del ojo del huracán.

Por qué se establecen estas parcelas. Bueno, porque se quería saber qué tan grande era la recuperación del bosque dependiendo de qué tan lejos estaba del centro del huracán. Las primeras parcelas medían $100 \times 10 \mathrm{~m}$, pero ya para 1994 se establecen parcelas de 50 x 30 metros, que son menores. Allí, las variables que se estudiaron fueron la altura y la circunferencia de todos los árboles que tuvieran más de 3.2 de diámetro y $10 \mathrm{~cm}$ de circunferencia

En cada árbol de la parcela se midieron estas dos variables con placas de aluminio permanente para dar continuidad a cada uno de los individuos de la parcela.

\section{RESPUESTA DEL BOSQUE DESPUÉS DEL JOAN}

Después del Joan, en cuanto a las estrategias de regeneración natural encontramos que el daño físico, para 1988, fue rápidamente superado; en cuatro meses, casi todas las especies estaban rebrotando y produciendo hojas.

\section{Estrategias del bosque}

Las estrategias básicamente fueron tres. En primer lugar, el bosque empezó a regenerase después de las plántulas y vástagos que habían quedado, pues los arboles pequeños son mas flexibles y pueden aguantar que un árbol grande les caiga encima y levantarse sin quebrarse. Esa fue la primera ruta de regeneración que se observó en este bosque: las plántulas y vástagos aprovechando el claro. El claro causado por el huracán era tan grande que lo primero que se esperaba que iban a venir eran las pioneras, tal como dice la teoría. Pero esto no pasó en este bosque, pues casi todas las especies encontradas eran de bosque primario y no secundario, es decir, había ausencia de especies pioneras. De esta forma se contradecía la teoría clásica de regeneración que dice que lo primero en regenerar son las especies pioneras.

La segunda estrategia de regeneración del bosque fueron los árboles en pie que estaban rebrotando de sus troncos a partir 
de $15 \mathrm{~m}$ de altura, entonces, ya tenían avanzado esos $15 \mathrm{~m}$, ya no tenían que partir de la semilla o de una plantita.

La tercera estrategia de recuperación fue la de aquellos arboles que se cayeron y que cada una de sus ramas se convirtió en un nuevo árbol. Esta estrategia es interesante, porque esto iba llevar a una segunda consecuencia que es el aumento en la densidad poblacional de las especies vegetales.

Si queremos hablar de la riqueza de especies que se observaron entonces, aquí tenemos los números de especies:

\section{Riqueza de especies luego de 11 años del paso del huracán Joan}

Total de arboles censados luego de 10 años del paso del huracán Joan:

Bodega $S=128$ en 1076 ind.

Fonseca $S=114$ en 1061 ind.

La Unión $S=79$ en 419 ind

Loma de Mico $S=82$ en 617 ind

Kurinwas $S=40$ y 63 en 232 A y 282 B

Fuente: Vandermeer et al, 2000

Science Vol 290, 788-791

\section{COMPORTAMIENTO DE ESPECIES PIONERAS TRAS EL PASO DEL HURACAN}

Como pueden comprobar, encontramos un bosque regenerándose de maravilla En La Bodega, después de casi 11 años del paso del huracán Joan pueden verse 128 especies representadas en 1,076 individuos. Para La Fonseca, que fue el siguiente sitio de muestreo, teníamos 144 especies y 1,061 individuos. Estos fueron los dos sitios iníciales y que presentaron la mayor riqueza en regeneración de toda el área afectada por el huracán. Luego La unión con 79 especies en 419 individuos: qué pasó aquí, hay que recordar que La Unión quedaba a $9 \mathrm{~km}$ de la trayectoria del huracán Joan y La Bodega quedaba a $14 \mathrm{~km}$, pues no hay correlación entre las distancia del centro de perturbación, con la riqueza que se estaba generando. La explicación de esto es que hubo un comportamiento distinto en cada sitio. Cuando queremos hablar de bosques tropicales podemos hacer generalidades, pero también podemos hacer particularidades y analizar cómo se comportan por sitio y, dentro de ese sitio, cómo se comportan algunas especies en el mismo sitio. En La Unión, que estaba muy cerca de un área de sotavento, entonces, la posible respuesta es que esta barrera física impidió que el daño fuera mayor en este sitio, y por lo tanto, este bosque está más estable por un proceso de competencia menos fuerte que el que había en La Bodega. De manera general, entonces, algunas especies que antes del huracán estaban constituidas por un solo individuo después regeneraron en dos, tres y hasta en seis individuos.

Para Kurinwas, que fue el área más lejana al ojo del huracán, vemos que no hay ningún incremento ni descenso. Ese bosque está en un estado de sucesión avanzada, es decir, en una etapa de bosque maduro, no

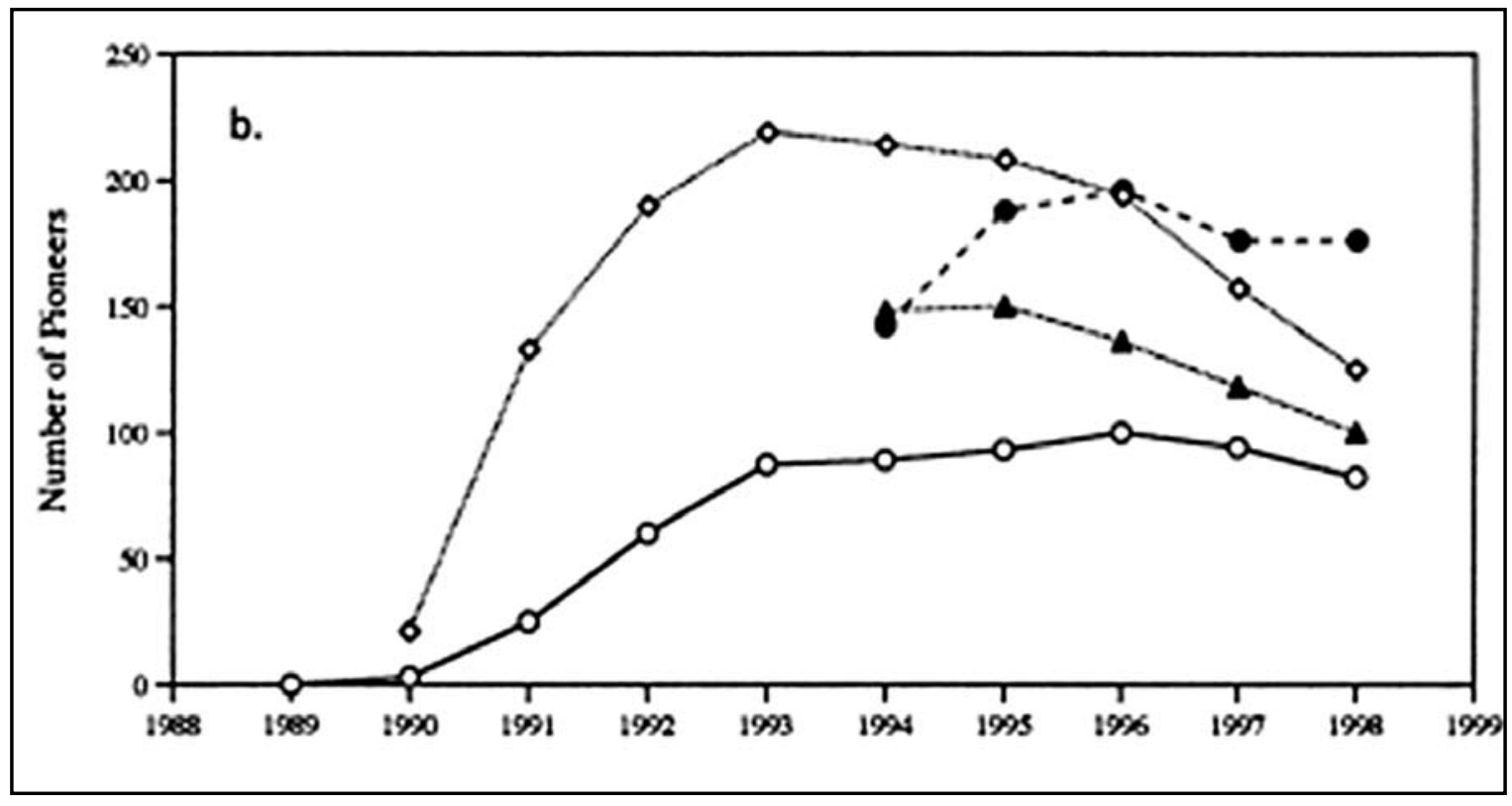


hay recambio de especies, ya está madura y esperando otro huracán.

También podemos ver cómo las especies pioneras tienen un ascenso increíble, pero no al inicio de la perturbación sino que ya comenzado el proceso de recuperación del bosque. Entonces, no estamos hablando de la teoría clásica de sucesión ecológica, que se autodenominó de regeneración directa. En realidad no es una regeneración directa en sí, porque tampoco están las especies clásicas pioneras. Hay una combinación de bosque maduro, pero también de nuevas especies pioneras que comenzaron a crecer, y éstas son las causantes de la riqueza del bosque que se refleja en los gráficos.

Luego, la teoría de sucesión pos huracanes. Una vez que está el bosque original y ocurre una perturbación, en este caso un huracán, pues se produce un daño físico al bosque. Y tenemos entonces que las especies, los árboles, están respondiendo a ese daño que se ha causado.

Luego de esas estrategias de recuperación, pues tenemos un bosque en construcción o reconstrucción de bosques. Estas especies empiezan a crecer. También está un segundo dosel, el más cerrado, que está compuesto por aquellas especies de plántula y vástagos que estuvieron creciendo. Después tenemos la parte del auto-raleo, que es cuando la competencia comienza a ser muy fuerte, puesto que los espacios de luz se estrechan y algunas especies empiezan a morir y otras a dominar el dosel superior.

Para el 2000 tenemos una estructura vertical casi homogénea, donde ya casi todos están a la altura, casi completamente recuperado el bosque. Por qué decimos que está casi recuperado, pues porque existen claros y, por lo tanto no puede llamarse maduro a este bosque. Para el 2002, catorce años después de la perturbación, pues tenemos una composición florística del bosque completamente recuperada. Ya están todas las especies que había antes del huracán, el bosque tiene una estructura vertical y horizontal más o menos parecida al estado en que estaba antes del huracán Joan. Por lo tanto se llega a la conclusión de que el bosque estudiado en la RAAS en solo catorce años se encuentra recuperado. Se necesitaron catorce años para que el bosque volviera a ser el mismo.

\section{Conclusiones}

La regeneración directa del bosque, en contraste de las predicciones de la teoría de sucesión ecológica, fueron evidenciadas en este estudio demostrando que no todos los bosques responden igual, porque los organismos vivos no todos respondemos igual frente a las perturbaciones, así mismo los bosques.

Los huracanes, entonces, podrían ser los responsables de un aumento temporal de la riqueza y la densidad de especies de árboles (Vandermeer, 1996). La riqueza de especies arbóreas resultante está relacionada con la magnitud del daño sufrido por el bosque.

La hipótesis inicial era que la recuperación del bosque estaba relacionada a la distancia del ojo del huracán. Aquí se encontró que no es la distancia, sino la magnitud del daño. La buena noticia es que el bosque perturbado sólo necesita 14 años, después del paso de un huracán, para recuperarse y alcanzar el estado inicial previo al huracán.

Entonces podemos pensar que la recuperación de un bosque es rápida, Pero lo que pasa es que la matriz en que están inmersos esos parches que fueron estudiados ya han cambiado mucho después de veinte años. Entonces, no podemos asumir que de aquí a veinte años la recuperación de un bosque perturbado es rápida, pero aún no sabemos con qué velocidad se recuperarán los bosques sometidos a otros tipos de perturbación, como el despale y los efectos causados por la agricultura migratoria, de monocultivo, de pasto, de tumba y quema. Hemos visto que se recuperan, pero no a la velocidad que lo hacen luego de un huracán. Lo importante es saber que lo que está afectando más a la naturaleza no son las perturbaciones naturales, que son parte de la dinámica de los ecosistemas que, hasta cierto punto, son necesarias a veces, sino que lo que más daña es la forma en que estamos explotando nosotros los recursos, llámense bosques o ecosistemas acuáticos.

Debemos dejar de ver el bosque únicamente como el proveedor de ingresos económicos, para pasarlo a ver en su dimensión ecológica y adaptarnos a esa dimensión ecológica de los recursos.

Muchas gracias 